\title{
Pain-Related Endurance, Fear-Avoidance and Somatosensory Sensitivity as Correlates of Clinical Status after Lumbar Disc Surgery
}

\author{
Sabine Melanie Held ${ }^{1}$, Roman Rolke ${ }^{2}$, Rolf-Detlef Treede ${ }^{3}$, Kirsten Schmieder ${ }^{4}$, Zohra Karimi ${ }^{1}$, \\ Sigrid Christa Sudhaus ${ }^{1}$ and Monika Ilona Hasenbring ${ }^{1, *}$
}

\begin{abstract}
${ }^{I}$ Department of Medical Psychology and Medical Sociology, Ruhr-University, Bochum, Germany; ${ }^{2}$ Department of Palliative Care, University of Bonn, Germany and Department of Neurology, University Medical Center, Johannes Gutenberg-University, Mainz, Germany; ${ }^{3}$ Neurophysiology, Center for Biomedicine and Medical Technology, Mannheim, Germany; ${ }^{4}$ Clinic of Neurosurgery, Knappschaftskrankenhaus, Bochum, Germany
\end{abstract}

\begin{abstract}
Most pain and disability variance in patients with low back pain still remains unexplained. The aim of this study was to enhance the degree of explained variance by including measures of pain and tactile sensitivity as well as pain-related endurance and fear-avoidance responses. Thirty-six post lumbar disc surgery patients completed psychometric questionnaires (Avoidance-Endurance Questionnaire, Fear-Avoidance Beliefs Questionnaire, Beck Depression Inventory) and underwent quantitative sensory testing (QST) with measures of pain (pressure (PPT) and mechanical pain threshold) and tactile sensitivity (MDT). Bivariate correlations and hierarchical multiple regression analysis were computed. In addition to the contribution of fear-avoidance responses, pressure pain sensitivity and endurance behavior significantly contributed to explanations of pain variance, whereas disability was primarily predicted by fear-avoidance. While all psychological variables and MDT were positively related to pain or disability, PPT was negatively related to pain. The regression model accounted for $69 \%$ of the variance in back pain intensity and $68 \%$ of the variance in disability. Tactile hypaesthesia was related to increased clinical pain. Pain-related endurance responses and pressure pain hyperalgesia were significant additional predictors for pain, but not for disability. These findings are compatible with generalized disinhibition via descending pathways and a general inhibition of tactile acuity by ongoing pain.
\end{abstract}

Keywords: Low back pain; endurance responses; fear-avoidance responses; quantitative sensory testing; sensory sensitivity.

\section{INTRODUCTION}

A considerable proportion of patients with disc herniation reports persistent pain and pain-related disability after primary lumbar disc surgery [1]. While most of these patients have no identifiable structural cause (e.g., structural abnormalities of the spinous elements) explaining their pain, a number of demographic factors [2] as well as psychosocial factors [3] are known to be important in maintaining pain and disability. However, multilevel models of data analysis, including structural, demographic and psychosocial factors as predictive variables, still account for a limited amount of the variance in clinical outcome. The investigation of new potential predictive variables will increase the explained variance in clinical outcomes and lend major insight towards understanding the processes of multiple determination of chronic low back pain (CLBP).

Psychosocial predictors, including depression, high job distress and fear-avoidance-related responses (FAR) to pain (e.g., catastrophizing, fear of pain and avoidance behavior), have been investigated extensively [3-6]. Recent research revealed evidence for an additional pathway involving endurance-related responses (ER); responses include conscious

\footnotetext{
*Address correspondence to this author at the Department of Medical Psychology and Medical Sociology, Ruhr-University of Bochum, Universitätsstraße 150, 44780 Bochum, Germany; Tel: ++49 23432 25439;

Fax: ++49 23432 14203; Email: monika.hasenbring@rub.de
}

suppression of pain-related thoughts and behavioral endurance despite severe pain, which leads to chronic pain via physical overload $[7,8]$. Although initial prospective studies have shown that ERs are additional predictors of pain [9, 10], scant empirical evidence considers the role of ER in explanations of variance of pain and especially disability.

Furthermore, recent research suggests that the integration of potential neurobiological predictors within multilevel models may indicate mechanisms of central plasticity. For example, the characteristics of the somatosensory phenotype of CLBP patients, such as increased pain sensitivity or reduced tactile function, may be of special interest [11]. Measures of individual pain thresholds under experimental pressure have been shown to account for additional unique variance in pain intensity and disability in CLBP [12]. A common phenomenon among CLBP patients is a feeling of numbness in painful body regions; thus, reduced tactile function may represent another characteristic of somatosensory phenotype that accounts for unique variance in clinical pain [13-15]. Tactile hypaesthesia following nociceptive stimulation has been reported by Geber et al. [16] using mechanical detection thresholds (MDT).

The present study aims to increase the proportion of the explained variance in clinical pain and disability by introducing new measures of behavior and neurobiological function that shed additional light on the complex interaction of psychological, social and biological factors involved in pain. 
The specific goals of this study are the following: 1. Explore the relationship between pain and tactile sensitivity and clinical back pain as well as disability. 2. Explore the relationship between pain-related ERs, clinical pain and disability, in addition to FAR and depression. 3. Investigate whether ER and measures of pain and tactile sensitivity (other than the well-known predictors) account for unique variance in multilevel models and increase the proportion of explained variance in clinical pain and disability.

\section{MATERIALS AND METHODOLOGY}

\subsection{Participants}

Documentation from a total of 150 patients with ages ranging from $20-65$ years was selected from the archives of the Department of Neurosurgery of the Knappschaftskrankenhaus Bochum, Germany and screened. Patients who had underwent a single lumbar disc surgery at least 6 and no more than 24 months prior to the start of the study and understood German met our inclusion criteria. Participants were additionally screened to exclude conditions that could affect somatosensation (e.g., diabetes, neuropathy, psychiatric disorders and current medical pain treatment). No current addresses were available for 73 patients, and 18 patients did not agree to participate. The medical examination reports of 6 patients were insufficiently detailed for study inclusion. Because of the exclusion criteria, 12 patients were omitted. Out of the 41 remaining patients, 4 were dropped due to incomplete data, and 1 was omitted because of circulatory insufficiency during testing. In total, measurements from 36 subjects were used for the analysis. All subjects were paid for participation and gave written informed consent. The study was conducted in cooperation with the German Research Network on Neuropathic Pain (DFNS) and was approved by the University Ethics Committee of the ChristianAlbrechts University in Kiel, Germany. The study was conducted in accordance with guidelines for good clinical practice as well as the Declaration of Helsinki.

\subsection{Procedures}

One of the authors telephoned patients fulfilling the criteria described above and invited them to participate in this study. The 3-part study was conducted at Bochum University. First, subjects participated in a short standardized physical evaluation with a general practitioner to obtain information about the biomedical status of the subjects. Next, self-report data were obtained using the research version of the Telemedical Patient-Diagnostics-System (TPDS), a personal computer-based self-report instrument [17] that included sociodemographic (gender and age), detailed painrelated and standardized psychometric questionnaires. The individual somatosensory phenotype was determined using quantitative sensory testing (QST).

\subsection{Measures}

\subsubsection{Description of the Sample}

For sample description, the date of disc surgery was documented to calculate the time between surgery and testing. Additionally, the Chronic Pain Grade (CPG) [18] was assessed to describe the severity of the subjects' chronic back pain. We used the German version of the chronic pain grade questionnaire, which was found to be reliable and valid in primary care back pain patients [19]. Adaptation of the original version's scoring rules resulted in a 5-point grading system: grade 0 stands for pain free, grade I stands for low disability and low pain intensity, grade II stands for low disability and high pain intensity, grade III stands for high disability and moderately limiting, and grade IV stands for high disability and severely limiting.

\subsubsection{Clinical Status: Back Pain Intensity and Pain- Related Disability}

Participants were asked to rate their average back pain intensity during the last 3 months by means of a visual pain rating scale; " 0 " stands for no pain, and " 10 " stands for maximum pain intensity. The patients completed the Pain Disability Index (PDI) [20], which is a 7-item inventory that asks the respondent to rate the perceived degree to which pain leads to disability in 7 areas of daily living: family/home responsibilities, recreation, social activity, occupation, sexual behavior, self-care and life-support activity. Each item score ranges from 0 (no disability) to 10 (total disability). Thus, the total PDI score ranges from 0 to 70 . This study used the German version of the PDI, which was found to be valid and reliable (coefficient alpha $=.88$ ) in chronic pain patients [21].

\subsubsection{Demographic Variables and Biomedical Status}

Gender and age were treated as demographic variables. The degree of lumbar forward flexion (lumbar flexibility) acted as an indicator for participant biomedical status. An inclinometer (Dr. Rippstein, Switzerland) was used for measurement. The first recording was conducted by holding the measuring instrument on the patient's spine at T12/L1 while in an erect position. With the inclinometer on T12/L1, the subjects were asked to bend forward and reach the fingertips of both hands as far toward their toes as possible while keeping their knees straight [22]. Lower degrees of lumbar forward flexion were taken as an indication of poor biomedical status.

\subsubsection{Depressive Symptoms}

The Beck Depression Inventory (BDI) is a 21-item selfreport measure assessing the incidence of various symptoms of depression (e.g., sad mood, sleep disturbance). This measure has demonstrated excellent reliability and validity as well as the ability to discriminate depressed from nondepressed pain patients $[23,24]$. The current study used the German version from Hautzinger [25], which is comparable with the English version. BDI total scores ranged from 0 to 63 points, with higher scores indicating more severe depressive symptoms.

\subsubsection{Somatosensory Phenotype}

The subjects underwent the quantitative sensory testing (QST) protocols as standardized by the German Research Network on Neuropathic Pain to measure their somatosensory phenotype [26]. Stimuli were applied to the lumbar musculus erector spinae next to the affected spinal segment (test area, TA) and the hand ipsilateral to the back pain area (intraindividual control area, ICA).

The pressure pain threshold (PPT) was measured by a handheld electronic pressure algometer (Somedic AB, Hörby, Sweden) with a contact area of $1 \mathrm{~cm}^{2}$ and a pressure 
application range from $0-2000 \mathrm{kPa}$. The intended rate of pressure increase $(50 \mathrm{kPa} / \mathrm{s})$ and the actual pressure applied were shown on a display. The pressure pain threshold was assessed with 3 series of ascending pressure intensities, and the final threshold was calculated as the mean of the 3 thresholds. The measurement was taken in the painful area of the musculus erector spinae and the thenar muscle of the accordant hand. A set of seven standardized and calibrated pinprick stimulators with flat contact areas $0.25 \mathrm{~mm}$ in diameter was used to assess the mechanical pain threshold (MPT). The stimulators exerted forces between 8 and 512 $\mathrm{mN}$ [27]. A total of 5 series of ascending and descending stimulus intensities (method of limits) were conducted, and the final threshold was the geometric mean of these intensities. The application was conducted on the musculus erector spinae and the back of the hand. Again, the "method of limits" was used to measure the mechanical detection threshold (MDT). Twelve standardized von Frey filaments were exerted between 0.25 and $512 \mathrm{mN}$ of force on the musculus erector spinae and the back of the hand. Each filament was provided with a rounded contact area $\left(<1 \mathrm{~mm}^{2}\right)$. The geometric mean of the intensities was calculated for the final threshold.

\subsubsection{Fear-Avoidance-Related Responses to Pain}

The "Avoidance of Social Activities Scale" (ASAS) of the Kiel Pain Inventory (KPI, a precursor of the AvoidanceEndurance Questionnaire, AEQ) [28] and the two workrelated scales of the German version of the Fear-Avoidance Beliefs Questionnaire (FABQ-D) [29] were used to measure subject fear-avoidance responses to pain. The ASAS is a 9item scale of the KPI that describes finishing or avoiding social activities due to low back pain (e.g., "I avoid getting together with others."). Cronbach's $\alpha$ was 0.91 . Patients used a 7-point Likert scale (never (0) to always (6)) to indicate frequency of thoughts and actions during pain experiences in the previous 14 days. Higher scores indicate a higher degree of self-reported fear-avoidance behavior.

The German version of the Fear-Avoidance Beliefs Questionnaire (FABQ-D) [30] consists of 3 subscales. The two work-related subscales explained high variance in the questionnaire (55.2\%) [29]. We used these subscales in our study. The scale "Causation by work" is a 6-item scale assessing respondent belief that back pain was caused by work (e.g., "My work could harm my back."). The scale "Prognosis work" is a 5 item-scale that describes the belief in a poor prognosis for returning to work (e.g., "I don't think that I could return to my regular job during the next 3 months."). Scores for each item range from 0 (not at all agree) to 6 (exactly agree). The German version of the FABQ was found to be reliable (Cronbach's $\alpha=.87$ ) in back pain patients.

\subsubsection{Endurance-Related Responses to Pain}

Subscales of the AEQ [31] were used to measure the pain-related endurance responses in the sample. The "Thought Suppression Scale" (TSS) consists of four items (e.g., "Pull yourself together") and describes the desire to avoid thinking about pain and attempts to suppress painrelated emotions and pain sensations. The "Behavioral Endurance Scale" (BES) of the AEQ is an 11-item scale that focuses on coping efforts aimed at finishing all activities in spite of severe back pain (e.g., "I keep all appointments even though I don't feel well.”). Patients used a 7-point Likert scale (never (0) to always (6)) to indicate frequency of thoughts and actions during pain experiences in the previous 14 days. Higher scores indicate a higher number of selfreported endurance cognitions and behavior. Internal consistency for the BES scale is reported with Cronbach's $\alpha=.83$ and $\mathrm{r}=.80$ for TSS [31].

\subsection{Statistical Analysis}

We assessed means, standard deviations (SD) and medians of variables as required for sample description. Scores of the psychometric measures KPI and AEQ are presented as the mean item scores, and the FABQ, PDI and BDI scores are shown as the mean sum scores. For descriptive and comparative purposes, we show decadal logarithmically transformed and retransformed raw data for PPT, MPT and MDT. To meet the requirements for further statistical analysis, decadal log-transformed data for MPT and MDT were used when the distributions for these parameters were skewed and differed significantly from the normal distribution as tested with a Kolmogorov-Smirnov test [32]. Bivariate Pearson's or Spearman's correlations (when required) were conducted to demonstrate relations between independent variables, such as gender and age (demographics); lumbar flexibility (biomedical status); BDI (depressive symptoms); PPT-ICA/ -TA, MPT-ICA/ -TA and MDT-ICA/ TA (somatosensory phenotype, QST); ASAS, FABQ-work and FABQ-prognosis (fearavoidance-related pain responses); and TSS and BES (endurance-related pain responses). The bivariate correlation between back pain intensity and pain-related disability (outcome variables) and each independent variable was studied by Pearson's or Spearman's correlations.

Finally, hierarchical multiple regression analysis for the two outcome variables was conducted. The independent variables were forced into regression analysis block by block. The entry of independent variables was performed in the following consecutive blocks: 1. demographics, 2. biomedical status, 3. depressive symptoms, 4. somatosensory phenotype, 5 . fear-avoidance-related responses and 6 . endurance-related responses. This hierarchical blockwise procedure allows for assessment of the significance of the explained variance $\left(R^{2}\right)$ increase attributable to the addition of each variable or block of variables into the equation. The entry method was chosen within blocks. Multicollinearity was checked at each step. Data were analyzed using the Statistical Package for the Social Sciences, SPSS 17.0 for Windows.

\section{RESULTS}

\subsection{Sample Characteristics}

Characteristics of the resulting sample are presented in Table 1. The total group of 36 participants $(18$ men, 18 women) had a mean age of 47.3 years ( $\mathrm{SD}=12.0$ years). On average, the disc surgery was performed 64.9 weeks $(\mathrm{SD}=$ 12.4 weeks) before testing. Using classification by Von Korff et al., the majority (58.3\%) of the group showed Chronic Pain Grade I [18]. These data are comparable with previous research data that examined patients after a first lumbar disc surgery [7]. In our sample, back pain intensity during the past three months averaged 2.64 on a 10-point rating scale (SD 2.2; Mdn 2.5). The mean PDI sum score 
Table 1. Patient Characteristics $(N=36)$

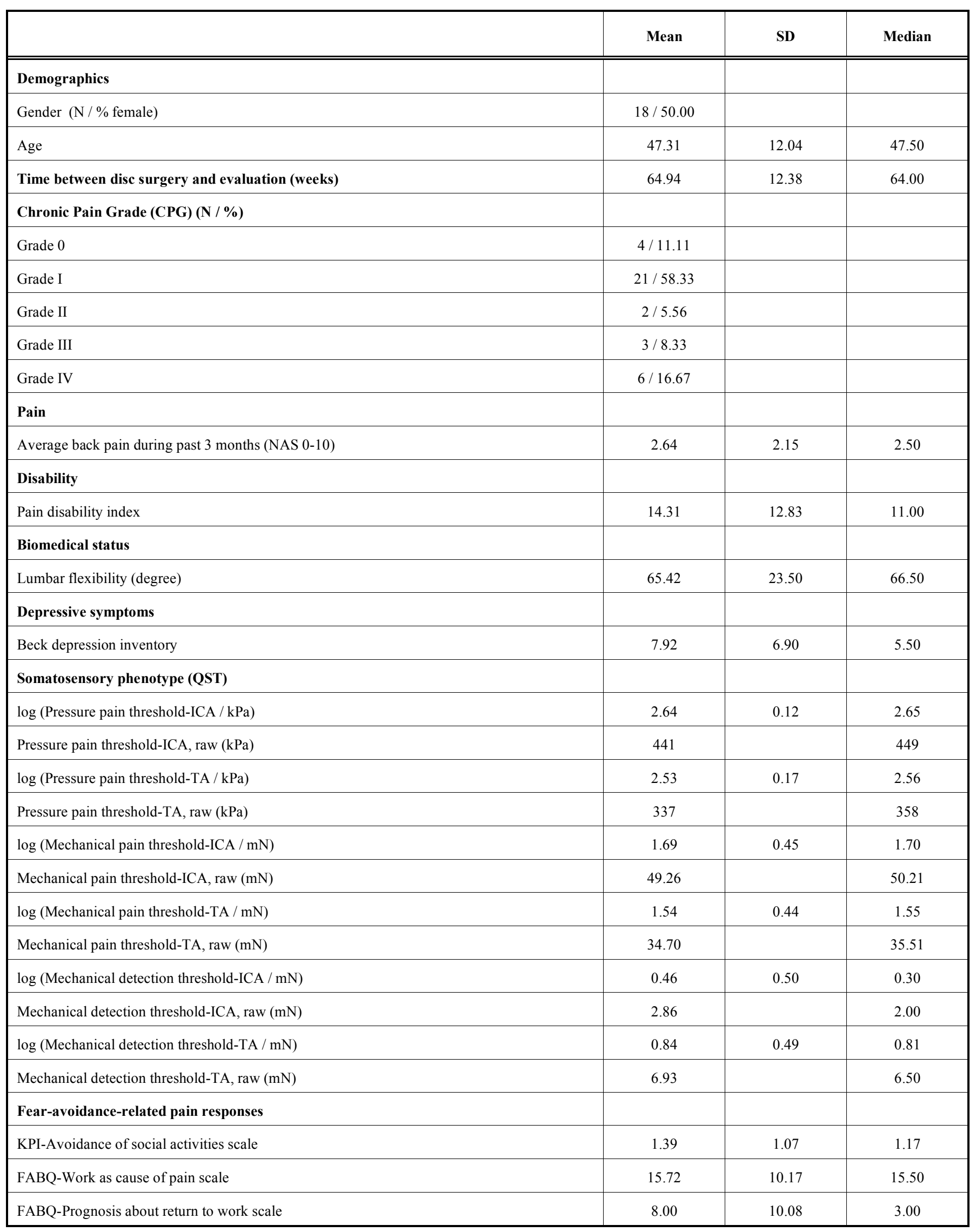


(Table 1) contd....

\begin{tabular}{|c|c|c|c|}
\hline & Mean & SD & Median \\
\hline \multicolumn{4}{|l|}{ Endurance-related pain responses } \\
\hline AEQ-Thought suppression scale & 3.06 & 1.69 & 3.25 \\
\hline AEQ-Behavioral endurance scale & 3.12 & 0.86 & 3.18 \\
\hline
\end{tabular}

CPG, Chronic pain grade; Grade 0, no pain; Grade I, low disability, low pain intensity; Grade II, low disability, high pain intensity; Grade III, high disability, moderate limiting; Grade IV, high disability, severe limiting; QST, Quantitative sensory testing; ICA, intraindividual control area (hand); TA, test area (back); log, values decadal logarithmically transformed; raw, retransformed to values with original unit; KPI, Kiel pain inventory; FABQ, Fear-avoidance beliefs questionnaire; AEQ, Avoidance-endurance questionnaire; TSS, Thought suppression scale; BES, Behavioral endurance scale.

was 14.3 (SD 12.8; Mdn 11.0). The distributions for the dependent and independent variables (including the transformed data for MPT and MDT) did not differ significantly from the normal distribution when examined by a Kolmogorov-Smirnov test.

\subsection{Bivariate Correlations}

Table 2 shows the results of the analysis of the intercorrelations between the independent variables. The correlation coefficients between independent variables and dependent variables (back pain intensity during the past three months and pain-related disability) and their significances are shown in Table 3.

\subsubsection{Back Pain Intensity}

Back pain intensity was significantly negatively correlated with the degree of lumbar flexibility (biomedical status) $(\mathrm{r}=-0.41, p<0.01)$. There was a significant positive correlation between back pain intensity and depressive symptoms $(\mathrm{r}=0.58, p<0.001)$. For the QST data, the back pain intensity was significantly negatively correlated with the pressure pain threshold at the hand (ICA) $(\mathrm{r}=-0.29, p<$ $0.05)$. The back pain intensity was significantly positively correlated with the mechanical detection threshold on ICA ( $\mathrm{r}$ $=0.41, p<0.01)$ and on TA $(\mathrm{r}=0.40, p<0.01)$. There was a significantly positive correlation between back pain intensity and avoidance of social activities $(\mathrm{r}=0.47, p<0.01)$, the fear-avoidance belief that work causes clinical back pain and the belief in a poor prognosis for returning to work $(\mathrm{r}=0.43$, $p<0.01$ and $\mathrm{r}=0.30, p<0.05$, respectively). For endurancerelated variables, the back pain intensity was significantly positively correlated with thought suppression and behavioral endurance $(\mathrm{r}=0.43, p<0.01$ and $\mathrm{r}=0.30, p<0.05)$.

\subsubsection{Disability}

There was a significant negative correlation between disability and the degree of lumbar flexion $(\mathrm{r}=-0.60, p<$ $0.001)$. Disability was significantly positively correlated with depressive symptoms $(\mathrm{r}=0.46, p<0.01)$. With respect to the QST variables, disability showed a trend toward a positive relation to the MDT of the hand and the back $(\mathrm{r}=0.25, p=$ 0.075 and $\mathrm{r}=0.25, p=0.067$, respectively). No association was found between disability and pain sensitivity measures. Among the fear-avoidance-related variables, avoidance of social activities $(\mathrm{r}=0.57, p<0.001)$, the fear-avoidance belief that work causes clinical back pain and the belief in a poor prognosis for returning to work $(\mathrm{r}=0.43, p<0.01$ and $\mathrm{r}$ $=0.60, p<0.001$, respectively) showed positive correlations with disability. Among endurance-related variables, disability trended toward a significant positive correlation with thought suppression $(\mathrm{r}=0.27, \mathrm{p}=0.056)$.

\subsection{Hierarchical Regression Analyses}

Two hierarchical multiple regression analyses were calculated with back pain intensity and disability as dependent variables. All variance inflation factors (VIF) ranged from 1.3 to 2.9 , indicating no multicollinearity concerns [33]. If the increase in explained variance $\left(R^{2}\right)$ for any block of predictor variables was significant, an analysis of the regression coefficients $(\beta)$ for each variable in that block revealed which variable was responsible for the significant increase in $R^{2}$. There were high intercorrelations between QST data on ICA and on TA (r-values ranging from 0.44 to $0.59, p<$ 0.01 ), and thus, we used ICA thresholds for regression analyses to reduce the number of independent variables.

\subsubsection{Back Pain Intensity}

Table 4 summarizes the results of the regression analysis for back pain intensity. First, patient demographic variables (gender and age) were entered into the regression equation. These variables accounted for only $1 \%$ of the variance in clinical back pain. The change in $R^{2}$ was $19 \%$ with each addition of the biomedical status variables lumbar flexibility and depressive symptoms to the equation. The set of somatosensory phenotype variables (PPT-ICA, MPT-ICA, MDTICA) accounted for $10 \%$ of the variance. Next, the set of fear-avoidance variables was entered into the regression equation, resulting in a $14 \%$ change in $R^{2}$. Finally, the set of endurance-related pain response variables was entered and accounted for $7 \%$ of the variance in back pain intensity. The final regression model $\left(\mathrm{F}_{12,23}=4.178, p \leq 0.01\right)$ accounted for $69 \%$ of the variance in clinical back pain. The standardized regression coefficients ( $\beta$-weights) were analyzed to assess which single variables were responsible for significant increases in explained variance. When all the variables were entered into the regression equation, only behavioral endurance $(p<0.05)$ and pressure pain threshold at ICA $(p<0.05)$ had significant unique contributions to the explanation of variance in back pain above the effects of the previously entered variables. Thus, patients with stronger behavioral endurance responses to back pain or high-pressure pain sensitivity had more intense back pain than patients who did not. There was also a trend $(p=0.058)$ for patients with the fear-avoidance belief "work causes pain" to report higher levels of back pain. 
Table 2. Intercorrelations Between Independent Variables

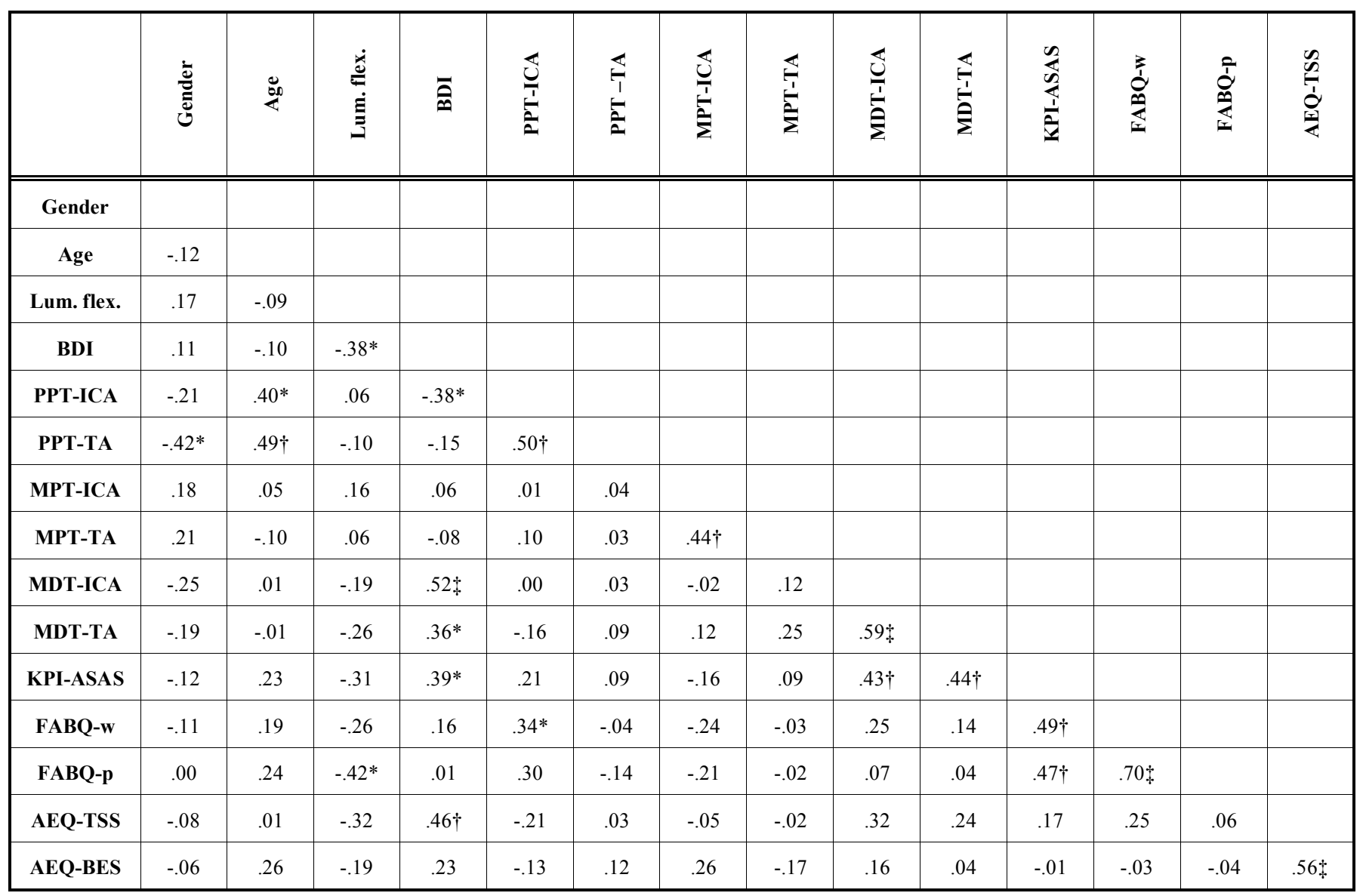

${ }^{*} p \leq 0.05 ; \dagger p \leq 0.01 ; \dagger p \leq 0.001$. Lum. flex., lumbar flexibility; BDI, Beck depression inventory; PPT, Pressure pain threshold; ICA, intraindividual control area (hand); TA, test area (back); MPT, Mechanical pain threshold; MDT, Mechanical detection threshold; KPI, Kiel pain inventory; ASAS, Avoidance of social activities scale; FABQ, Fear-avoidance beliefs questionnaire; FABQ-w, Work as cause of pain scale; FABQ-p, Prognosis about return to work scale; AEQ, Avoidance-endurance questionnaire; TSS, Thought suppression scale; BES, Behavioral endurance scale.

\subsubsection{Disability}

A summary of the regression analysis for pain-related disability is presented in Table 5. We entered the variables or blocks of variables into the disability regression equation in the same order as the back pain intensity regression. Patient demographic variables (gender and age) accounted for less than $1 \%$ of the variance in clinical back pain. In the next step, lumbar flexibility introduction into the equation increased $R^{2}$ by $36 \%$. Depressive symptoms and the set of somatosensory phenotype variables were entered in the following two steps and produced a $6 \%$ change in $R^{2}$ for each. With the addition of the set of fear-avoidance variables, the change in $R^{2}$ was $20 \%$. The set of endurance-related pain response variables was entered last and accounted for less than $1 \%$ of variance in disability. The final regression model $\left(\mathrm{F}_{12,23}=4.012, p<0.01\right)$ accounted for $68 \%$ of the variance in pain-related disability. When all variables were entered into the regression equation, only the fear-avoidance variable belief in a poor prognosis for returning to work $(p<0.05)$ had a significant unique contribution to the explanation of variance in disability above the effects of the previously entered variables. Patients who had a stronger belief in a poor prognosis for returning to work reported more disability than patients who did not have this belief.

\section{DISCUSSION}

This study aimed to increase the proportion of explained variance in back pain intensity and pain-related disability by introducing new measures of somatosensory tenderness (QST) and pain-related behavior. The results indicated that the prediction of pain intensity was significantly improved by pain sensitivity and pain-related endurance responses (ER) above and beyond the contribution of fear-avoidance responses (FAR). In contrast, disability was primarily predicted by FAR, whereas somatosensory phenotype and endurance had minor importance. Examination of the standardized regression coefficients of the final models indicated that the initially high impact of the variables biomedical status, depression and tactile sensitivity was reduced in the context of all included variables; this result may be due to high intercorrelations with FAR and ER variables.

\subsection{Low-Back Pain and Mechanical Pain Sensitivity}

Clauw and colleagues [12] were the first to demonstrate that after controlling for demographic, structural and psychological variables, pain sensitivity measured by pressure over painful and control areas accounts for additional unique variance in pain and self-reported physical function. The sample that Clauw and colleagues examined consisted of patients with severe chronic low back pain and some symptoms of 
Table 3. Correlation Coefficients Between Outcome Variables and Independent Variables

\begin{tabular}{|c|c|c|c|c|}
\hline & \multicolumn{2}{|c|}{ Pain (NAS) } & \multicolumn{2}{|c|}{ Disability (PDI) } \\
\hline & $\mathbf{r}$ & $\mathbf{p}$ & $\mathbf{r}$ & $\mathbf{p}$ \\
\hline Gender & 0.07 & 0.353 & 0.13 & 0.227 \\
\hline Age & -0.08 & 0.320 & 0.03 & 0.426 \\
\hline Lumbar flexibility & -0.41 & 0.006 & -0.60 & $<0.001$ \\
\hline \multicolumn{5}{|l|}{ Depressive symptoms } \\
\hline BDI & 0.58 & $<0.001$ & 0.46 & 0.002 \\
\hline Mechanical pain threshold-ICA & -0.24 & 0.084 & -0.06 & 0.357 \\
\hline Mechanical pain threshold-TA & -0.21 & 0.113 & 0.05 & 0.385 \\
\hline Mechanical detection threshold-ICA & 0.41 & 0.007 & 0.25 & 0.075 \\
\hline Mechanical detection threshold-TA & 0.40 & 0.007 & 0.25 & 0.067 \\
\hline \multicolumn{5}{|l|}{ Fear-avoidance responses } \\
\hline KPI-avoidance of social activities scale & 0.47 & 0.002 & 0.57 & $<0.001$ \\
\hline FABQ-work as cause of pain scale & 0.43 & 0.004 & 0.43 & 0.004 \\
\hline
\end{tabular}

NAS, Numerical analog scale (0 - 10), average back pain during past 3 months; PDI, Pain disability index; BDI, Beck depression inventory; ICA, intraindividual control area (hand); TA, test area (back); KPI, Kiel pain inventory; FABQ, Fear-avoidance beliefs questionnaire; AEQ, Avoidance-endurance questionnaire.

fibromyalgia. The results of our study indicate that measures of pain threshold are additional significant predictors in samples of patients 6 months after lumbar disc surgery.

Clauw and colleagues [12] reported that similar variables account for variance in back pain and physical function; however, our study additionally indicates a differentiation between these outcomes. After controlling for gender, age, biomedical status, depressive symptoms and behavioral endurance, pressure pain sensitivity accounted for significant additional variance in back pain intensity. In contrast, disability was independently predicted by the fear-avoidance belief in a poor prognosis for returning to work. We found significant negative bivariate associations between clinical pain and pressure pain thresholds in the hand. These results are in line with a recently reported generalized deep-tissue hyperalgesia in CLBP patients with an MRI-confirmed intervertebral disc herniation, which was measured by pressure pain thresholds in the infraspinatus and tibialis anterior muscles [34], and suggest a loss of descending inhibition or increase of descending facilitation as a generalized mechanism of increased pain sensitivity $[35,36]$.
In contrast to pain tenderness in a non-painful area, the present study did not show a significant relationship between clinical pain and pain sensitivity in the back for either pressure pain or pinprick pain. This result is contrary to former studies (e.g., [37]). One explanation may be that local sensitivity to pressure in deep muscles may be impaired following the lumbar disc surgery procedure (e.g., by scar tissue).

\subsection{Low-Back Pain and Tactile Sensitivity}

Concerning tactile sensitivity, bivariate correlations revealed significant positive associations between experimental tactile thresholds and back pain. Presently, different models exist to supply a background to interpret the relationship between reduced tactile function and increased clinical pain. Geber et al. [16] investigated patients with musculoskeletal pain and found support for their assumption that reduction of tactile function is a neurophysiological consequence of the activation of nociceptive pathways. The authors also found higher mean pain ratings associated with an increase in tactile hypaesthesia. Supporting this interpretation, Moseley [38] reported reduced tactile acuity in the area of usual pain 
Table 4. Summary of Hierarchical Regression Analysis for Clinical Back Pain Intensity (R2 of the Final Model = 0.69, F12, 23 Ratio for $R 2=4.178 \dagger$ )

\begin{tabular}{|c|c|c|c|}
\hline Step & Variables & $\beta^{\mathrm{a}}$ & $\mathbf{R}^{2}$ change \\
\hline \multirow[t]{2}{*}{1} & Demographics & & .013 \\
\hline & Age & -0.134 & \\
\hline 2 & Biomedical status & & .186 \\
\hline \multirow[t]{2}{*}{3} & Depressive symptoms & & .185 \\
\hline & BDI & 0.255 & \\
\hline 4 & Somatosensory phenotype (QST) & & .100 \\
\hline \multirow[t]{4}{*}{5} & Fear-avoidance-related pain responses & & .135 \\
\hline & KPI-avoidance of social activities scale & 0.235 & \\
\hline & FABQ-work as cause of pain scale & 0.383 & \\
\hline & FABQ-prognosis about return to work scale & -0.015 & \\
\hline \multirow[t]{3}{*}{6} & Endurance-related pain responses & & .066 \\
\hline & AEQ-thought suppression scale & -0.135 & \\
\hline & AEQ-behavioral endurance scale & $0.359 *$ & \\
\hline
\end{tabular}

a Standardized $\beta$ weights and significances are displayed from the final model.

$* p \leq 0.05, \dagger p \leq 0.01$. BDI, Beck depression inventory; ICA, intraindividual control area (hand); KPI, Kiel pain inventory; FABQ, Fear-avoidance beliefs questionnaire; AEQ, Avoidance-endurance questionnaire.

in CLBP patients compared to healthy controls when measured by tactile thresholds and a two-point discrimination threshold (TPD). Moseley suggested that tactile acuity training is an important component of CLBP treatment that may contribute to the reduction of pain and increase in function $[39,40]$.

A generalized impairment of interoception may further explain reduced tactile acuity [41]. Given the limited processing capacity of afferent signals [42], the competition-ofcues model of interoception [43] provides a cognitive explanation for reduced interoceptive acuity. A reduced interoceptive awareness associated with an increased awareness of external stimuli is hypothesized to play a role in cardiovascular diseases [44]. We suggest that a similar pathway may exist in patients suffering from back pain after lumbar disc surgery. Although highly speculative at the moment, reduced interoceptive acuity may lead to overuse and overload of the muscles, bones and ligaments known to induce and maintain low back pain (e.g., [45]).

\subsection{Low Back Pain and Pain-Related Fear-Avoidance and Endurance}

The present study revealed the unique predictive power of ER, even after controlling for depression and other painrelevant variables. The fear-avoidance belief that work causes pain (FABQ-work), self-reported prognosis for returning to work and self-reported avoidance behavior had positive relationships to pain and accounted for $14 \%$ of the variance in pain. This finding is in line with a number of cross-sectional and prospective studies showing that FARs are associated with pain (see $[3,5,46]$ for reviews). Rather new is the finding that, in addition to FAR, ERs, such as thought suppression and task persistence behavior, were positively related to clinical pain. With respect to the avoidance-endurance model of pain $[47,48]$, we suggest that task persistence behavior despite severe pain will lead to an overuse/overload of physical structures, causing repeated, minor damage to the muscles and ligaments and subsequent persistent pain. Prospective studies have suggested that high levels of physical activity are associated with an increased risk of lumbar radicular pain (see [49] for review). Thought suppression is linked to pain primarily via an increase in depression, mediated by a rebound phenomenon that causes feelings of failure and reduced self-efficacy [50]. The existence of pain coping strategies beyond FAR was previously shown with the use of several self-report measures of coping efforts. Rosenstiel and Keefe [51] investigated this issue with the Coping Strategies Questionnaire (CSQ), which ascertains how frequently chronic back pain patients use various coping strategies and how effective patients rate these strategies 
Table 5. Summary of Hierarchical Regression Analysis for Disability (R2 of the Final Model = 0.68, F12, 23 Ratio for R2 $=4.012 \uparrow$ )

\begin{tabular}{|c|c|c|c|}
\hline \multirow[t]{2}{*}{1} & Demographics & & 0.003 \\
\hline & Age & -0.155 & \\
\hline \multirow[t]{2}{*}{3} & Depressive symptoms & & 0.057 \\
\hline & BDI & 0.279 & \\
\hline 4 & Somatosensory phenotype (QST) & & 0.055 \\
\hline \multirow[t]{4}{*}{5} & Fear-avoidance-related pain responses & & 0.196 \\
\hline & KPI-avoidance of social activities scale & 0.269 & \\
\hline & FABQ-work as cause of pain scale & -0.140 & \\
\hline & FABQ-prognosis about return to work scale & $0.467 *$ & \\
\hline \multirow[t]{3}{*}{6} & Endurance-related pain responses & & 0.005 \\
\hline & AEQ-thought suppression scale & 0.107 & \\
\hline & AEQ-behavioral endurance scale & -0.044 & \\
\hline
\end{tabular}

${ }^{\text {a }}$ Standardized $\beta$ weights and significances are displayed from the final model.

$* p \leq 0.05, \dagger p \leq 0.01$. BDI, Beck depression inventory; ICA, intraindividual control area (hand); KPI, Kiel pain inventory; FABQ, Fear-avoidance beliefs questionnaire; AEQ, Avoidance-endurance questionnaire.

during their adjustment to chronic pain. The researchers found coping strategies that ignored pain sensations and increased activity levels were similar to the ER discussed within the AEM. Jensen et al. [52] developed an inventory to assess a wider variety of cognitive and behavioral pain coping strategies (Chronic Pain Coping Inventory, CPCI) compared with the CSQ. The authors categorized task persistence (e.g., "Did not let the pain affect what I was doing") as a wellness-focused coping strategy because this behavior was negatively associated with depression and pain-related distress. In contrast, our study did not reveal a negative association between behavioral endurance despite severe pain and depression. Our results indicate that this pain response is associated with a higher level of back pain intensity. However, our results did not reveal a negative effect of behavioral endurance on pain-related disability.

\subsection{Predictors of Disability}

While ER and QST variables significantly increased the proportion of variance explained in clinical pain, this finding was not evident in disability. For disability, the fearavoidance belief in a poor prognosis for returning to work was particularly important in our explanatory model, whereas structural indices, measures of somatosensory phenotype, depression and ER were less important in the explanation of disability. Good evidence supports the relationship between pain-related fear and disability (see [46] for review). In a group of female health care workers with LBP on 1 or more days during the previous 12 months, high fearavoidance beliefs increased the risk of missing work due to sickness one year later [53]. Our results are in accordance with findings on pain-related fear of movement/(re) injury or negative outcome expectancies as predictors for greater disability after lumbar disc surgery [54].

\subsection{Technical Considerations}

There are several study limitations. The sample size was inadequate to detect small effects of the predictor variables on the clinical outcomes of pain intensity and disability. The ratios between subjects and independent variables in the multivariate analyses may result in unstable regression coefficients. Replications using larger sample sizes are needed to shed light on the multi-determination of chronic clinical pain and disability. All somatosensory data are based on selfreport and potentially affected by variables we did not consider. The authors did not include other important factors, such as compensation status or personality factors, which may be predictive of pain and disability.

\section{CONCLUSION}

Using QST, it is possible to characterize patient sensitivity to different evoked painful and non-painful stimuli. The 
somatosensory phenotype (pressure pain hyperalgesia at the hand and tactile hypaesthesia at the hand and the lower back) as well as pain-related endurance behavior largely explained the intensity of ongoing pain. Although we need longitudinal studies investigating hypothesized predictors and outcomes over time to determine the direction of causation, we may conclude that pressure pain sensitivity as well as pain-related endurance behavior could be important additional diagnostic tools for clinical back pain patients. Distinguishing patient ER and FAR appears to be helpful for treatment. The effectiveness of cognitive-behavioral treatments in reducing disability was associated with reduced pain catastrophizing $[55$, 56] and pain-related fear [57, 58]. Cognitive restructuring and confrontation with the fearful situation are effective in reducing catastrophizing cognitions of patients with high FAR. In contrast, patients with high ER should be encouraged to accept the pain experience as a signal that indicates the importance of taking breaks in daily activities and reducing physical postures that cause a high load on muscles and the spine. Interventions including sensory monitoring [47], reduction of thought suppression and task persistent behavior might help these patients reach more flexible responses to pain [48].

\section{CONFLICT OF INTEREST}

There are no conflicts of interest. This study was supported by grant 01 EM 0114 of the BMBF within the DFRS (German Research Network on Back Pain) and 01 EM 0901 of the DFNS (German Research Network on Neuropathic Pain).

\section{ACKNOWLEDGEMENTS}

We thank Katja Burkhardt, Dr. Dirk Hallner and Heike Plaas for their contributions and helpful critiques. We also thank the subjects who participated in the study for their consent and cooperation.

\section{REFERENCES}

[1] Krämer J. Bandscheibenbedingte Erkrankungen. Stuttgart: Thieme 2006.

[2] Schmidt CO, Raspe H, Pfingsten M, et al. Back pain in the German adult population: prevalence, severity, and sociodemographic correlates in a multiregional survey. Spine 2007; 32(18): 2005-11.

[3] Linton SJ. A review of psychological risk factors in back and neck pain. Spine 2000; 25(9): 1148-56.

[4] Grotle M, Brox JI, Glomsrød B, Lønn JH, Vøllestad NK. Prognostic factors in first-time care seekers due to acute low back pain. Eur J Pain 2007; 11(3): 290-8

[5] Leeuw M, Goossens MEJB, Linton SJ, Crombez G, Boersma K, Vlaeyen JWS. The fear-avoidance model of musculoskeletal pain: current state of scientific evidence. J Behav Med 2007; 30(1): 7794.

[6] Peul WC, Arts MP, Brand R, Koes BW. Timing of surgery for sciatica: subgroup analysis alongside a randomized trial. Eur Spine J 2009; 18(4): 538-45.

[7] Hasenbring M, Plaas H, Fischbein B, Willburger R. The relationship between activity and pain in patients 6 months after lumbar disc surgery: do pain-related coping modes act as moderator variables? Eur J Pain 2006; 10(8): 701-9.

[8] Bousema EJ, Verbunt JA, Seelen HA, Vlaeyen JW, Knottnerus JA. Disuse and physical deconditioning in the first year after the onset of back pain. Pain 2007; 130(3): 279-86.

[9] Hasenbring M, Marienfeld G, Kuhlendahl MD, Soyka D. Risk factors of chronicity in lumbar disc patients. A prospective investigation of biologic, psychologic, and social predictors of therapy outcome. Spine 1994; 19(24): 2759-65.
[10] Grebner M, Breme K, Rothoerl R, Woertgen C, Hartmann A, Thomé C. Coping und Genesungsverlauf nach lumbaler Bandscheibenoperation. Schmerz 1999; 13(1): 19-30.

[11] Rolke R, Baron R, Maier C, et al. Quantitative sensory testing in the German Research Network on Neuropathic Pain (DFNS): standardized protocol and reference values. Pain 2006a; 123(3): 23143.

[12] Clauw DJ, Williams D, Lauerman W, et al. Pain sensitivity as a correlate of clinical status in individuals with chronic low back pain. Spine 1999; 24(19): 2035-41.

[13] Leffler AS, Hansson P, Kosek E. Somatosensory perception in patients suffering from long-term trapezius myalgia at the site overlying the most painful part of the muscle and in an area of pain referral. Eur J Pain 2003; 7(3): 267-76.

[14] Magerl W, Treede RD. Secondary tactile hypoesthesia: a novel type of pain-induced somatosensory plasticity in human subjects. Neurosci Lett 2004; 361(1-3): 136-9.

[15] Freynhagen R, Rolke R, Baron R, et al. Pseudoradicular and radicular low-back pain - a disease continuum rather than different entities? Answers from quantitative sensory testing. Pain 2008; 135(1-2): 65-74.

[16] Geber C, Magerl W, Fondel R, et al. Numbness in clinical and experimental pain - a cross-sectional study exploring the mechanisms of reduced tactile function. Pain 2008; 139(1): 73-81.

[17] Hasenbring M, Hallner D. Telemedizinisches Patienten-DiagnoseSystem (TPDS). Selbsterklärende PC-Lösung zur Analyse von Risikofaktoren der Chronifizierung von Rückenschmerzen. Dtsch Arztebl, 1999; 96: 49-50.

[18] Von Korff M, Ormel J, Keefe FJ, Dworkin SF: Grading the severity of chronic pain. Pain 1992; 50(2): 133-49.

[19] Klasen BW, Hallner D, Schaub C, Willburger R, Hasenbring M. Validation and reliability of the german version of the chronic pain grade questionnaire in primary care back pain patients. Psychosoc Med 2004; 1: Doc07.

[20] Pollard CA. Preliminary validity study of the pain disability index. Percept Mot Skills 1984; 59(3): 974.

[21] Dillmann U, Nilges $P$, Saile H, Gerbershagen HU. Behinderungseinschätzung bei chronischen Schmerzpatienten. Schmerz 1994; 8(2): 100-10.

[22] Waddell G, Somerville D, Henderson I, Newton M. Objective clinical evaluation of physical impairment in chronic low back pain. Spine 1992; 17(6): 617-28.

[23] Turner JA, Romano JM. Self-report screening measures for depression in chronic pain patients. J Clin Psychol 1984; 40(4): 909-13.

[24] Geisser ME, Roth RS, Robinson ME. Assessing depression among persons with chronic pain using the Center for Epidemiological Studies-Depression Scale and the Beck Depression Inventory: a comparative analysis. Clin J Pain 1997; 13(2): 163-70.

[25] Hautzinger M. The Beck Depression Inventory in clinical practice. Nervenarzt 1991; 62(11): 689-96.

[26] Rolke R, Magerl W, Campbell KA, et al. Quantitative sensory testing: a comprehensive protocol for clinical trials. Eur J Pain 2006b; 10(1): 77-88.

[27] Baumgärtner U, Magerl W, Klein T, Hopf HC, Treede RD. Neurogenic hyperalgesia versus painful hypoalgesia: two distinct mechanisms of neuropathic pain. Pain 2002; 96: 141-51.

[28] Hasenbring M. Das Kieler Schmerzinventar. Manual. Bern: Hans Huber 1994.

[29] Pfingsten M, Leibing E, Franz C, Bansemer D, Busch O, Hildebrandt J. Erfassung der ,fear-avoidance-beliefs“ bei Patienten mit Rückenschmerzen. Deutsche Version des „Fear-AvoidanceBeliefs Questionnaire“(FABQ-D). Schmerz 1997; 11: 387-95.

[30] Pfingsten M, Kröner-Herwig B, Leibing E, Kronshage U. Validation of the German version of the Fear-Avoidance Beliefs Questionnaire (FABQ). Eur J Pain 2000; 4(3): 259-66.

[31] Hasenbring MI, Hallner D, Rusu AC. Fear-avoidance- and endurance-related responses to pain: development and validation of the Avoidance-Endurance Questionnaire (AEQ). Eur J Pain 2009; 13: 620-8.

[32] Kosek E, Ordeberg G. Lack of pressure pain modulation by heterotopic noxious conditioning stimulation in patients with painful osteoarthritis before, but not following, surgical pain relief. Pain 2000; 88: 69-78.

[33] Stevens J. Applied multivariate statistics for the social sciences. Mahwah: Lawrence Erlbaum Associates, Inc.; 1996. 
[34] O'Neill S, Manniche C, Graven-Nielsen T, Arendt-Nielsen L. Generalized deep-tissue hyperalgesia in patients with chronic low-back pain. Eur J Pain 2007; 11(4): 415-20.

[35] Leffler AS, Hansson P, Kosek E. Somatosensory perception in a remote pain-free area and function of diffuse noxious inhibitory controls (DNIC) in patients suffering from long-term trapezius myalgia. Eur J Pain 2002; 6: 149-59.

[36] Giesecke T, Gracely RH, Clauw DJ, et al. Zentrale Schmerzverarbeitung bei chronischem Rückenschmerz. Hinweise auf verminderte Schmerzinhibition. Schmerz 2006; 5: 411-7.

[37] Farasyn A, Meeusen R. The influence of non-specific low back pain on pressure pain thresholds and disability. Eur J Pain 2005; 9(4): 375-81.

[38] Moseley GL. I can't find it! Distorted body image and tactile dysfunction in patients with chronic back pain. Pain 2008; 140(1): 239-43.

[39] Flor H, Elbert T, Knecht S, et al. Phantom-limb pain as a perceptual correlate of cortical reorganization following arm amputation. Nature 1995; 375(6531): 482-4.

[40] Mosely GL, Zalucki NM, Wieck K. Tactile discrimination, but not tactile stimulation alone, reduces chronic limb pain. Pain 2008; 137: 600-8.

[41] Craig AD. Human feelings: why are some more aware than others? Trends Cogn Sci 2004; 8(6): 239-41.

[42] Kahnemann D. Attention and effort. Englewood Cliffs: PrenticeHall, Inc.; 1973.

[43] Pennebaker JW. Psychology of physical symptoms. New York: Springer 1982.

[44] Kollenbaum VE. Interozeption kardiovaskulärer Belastung bei Koronarpatienten. Ein Beitrag zur Rehabilitation der koronaren Herzkrankheit. Frankfurt am Main: Peter-Lang 1990.

[45] Sbriccoli P, Solomonow M, Zhou BH, et al. Static load magnitude is a risk factor in the development of cumulative low back disorder. Muscle Nerve 2004; 29(2): 300-8.

[46] Vlaeyen JW, Linton SJ. Fear-avoidance and its consequences in chronic musculoskeletal pain: a state of the art. Pain 2000; 85(3): 317-32.

[47] Hasenbring M. Attentional control of pain and the process of chronification. In: Sandkühler J, Bromm B and Gebhart CF, Eds. Progress in brain research. Amsterdam: Elsevier 2000; vol. 129: pp. 525-34.
[48] Hasenbring M, Verbunt JA. Fear-avoidance and endurance-related responses to pain: new models of behavior and their consequences for clinical practice. Clin J Pain 2010; 26(9): 747-53.

[49] Shiri R, Karppinen J, Leino-Arjas P, et al. Cardiovascular and lifestyle risk factors in lumbar radicular pain or clinically defined sciatica: a systematic review. Eur Spine J 2007; 16: 2043-54.

[50] Wegner DM. You can't always think what you want: Problems in the suppression of unwanted thought. In: Zanna M, Ed. Advances in experimental social psychology. San Diego: Academic Press 1992; vol. 25: pp. 193-225.

[51] Rosenstiel AK, Keefe FJ. The use of coping strategies in chronic low back pain patients: relationship to patient characteristics and current adjustment. Pain 1983; 17: 33-44.

[52] Jensen MP, Turner JA, Romano JM, Strom SE. The chronic pain coping inventory: development and preliminary validation. Pain 1995; 60: 203-16.

[53] Jensen JN, Karpatschof B, Labriola M, Albertsen K. Do fearavoidance beliefs play a role on the association between low back pain and sickness absence? A prospective cohort study among female health care workers. J Occup Environ Med 2010; 52(1): 8590 .

[54] den Boer JJ, Oostendorp RAB, Beems T, Munneke M, Evers AWM. Continued disability and pain after lumbar disc surgery: the role of cognitive-behavioral factors. Pain 2006; 123: 45-52.

[55] Smeets RJEM, Vlaeyen JWS, Kester ADM, Knottnerus JA. Reduction of pain catastrophizing mediates the outcome of both physical and cognitive-behavioral treatment in chronic low back pain. J Pain 2006; 7: 261-71.

[56] Sullivan MJL, Ward LC, Tripp D, French DJ, Adams H, Stanish WD. Secondary prevention of work disability: community-based psychosocial intervention for musculoskeletal disorders. J Occup Rehabil 2005; 15: 377-92.

[57] Klaber Mofett JA, Carr J, Howarth EH. High fear-avoiders of physical activity benefit from an exercise program for patients with back pain. Spine 2004; 29: 1167-79.

[58] Woby SR, Watson PJ, Roach NK, Urmston M. Are changes in fear-avoidance beliefs, catastrophizing, and appraisals of control, predictive of changes in chronic low back pain and disability). Eur J Pain 2004; 8: 201-10.

Received: March 04, 2013

Revised: April 19, 2013

Accepted: April 29, 2013

(C) Held et al.; Licensee Bentham Open.

This is an open access article licensed under the terms of the Creative Commons Attribution Non-Commercial License (http://creativecommons.org/licenses/by-nc/3.0/) which permits unrestricted, non-commercial use, distribution and reproduction in any medium, provided the work is properly cited. 